\title{
Application Model of Modern Piano Education by Online Software of Everyone_Piano
}

\author{
Xuyan Wang ${ }^{1,}$ a * \\ ${ }^{1}$ Teacher's College, Beihua University, Jilin, China \\ a254519852@qq.com
}

Keywords: internet public opinions; Chinese language; construction; network monitoring Abstract. With the progress of science and technology, the APP software's were applied in piano teaching, such as Everyone_Piano. Everyone_Piano has been applied in modern piano teaching is very widely by online ways. Application results show that the application of Everyone_Piano has been catch the student attention easily to learn music literacy, more easily to professional music development.

\section{Introduction}

In recent years, the Internet and computer information technology as the representative of the rapid development of appropriate computer software and multimedia audiovisual equipment is becoming mature and function has become more diversified. Its application in the field of education, to the entire education sector has undergone enormous changes; especially the development of the piano education has played a prominent role in promoting. Multimedia technology has greatly improved the traditional model of piano education: a book, a piano, a teacher. Among lively in the classroom increases the video screen, the student's visual sensory irritation; adds realistic classic piano repertoire, teaching content, and students produce auditory stimuli. Multimedia Piano Teaching also makes the classroom atmosphere becomes more lively and active, has greatly improved the students' interest, thus contributing to piano teaching efficiency.

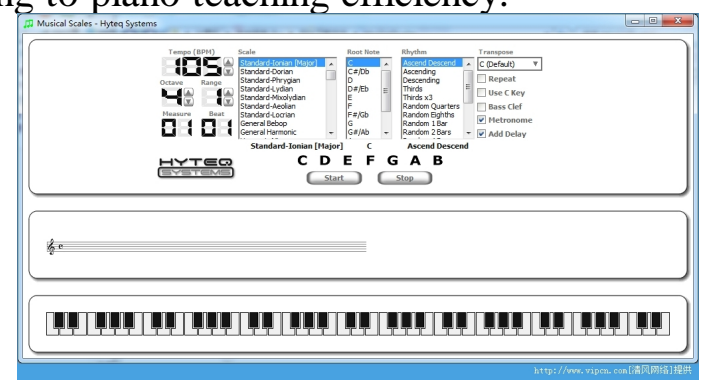

Fig.1 MidiSoft PlayPiano demo interface

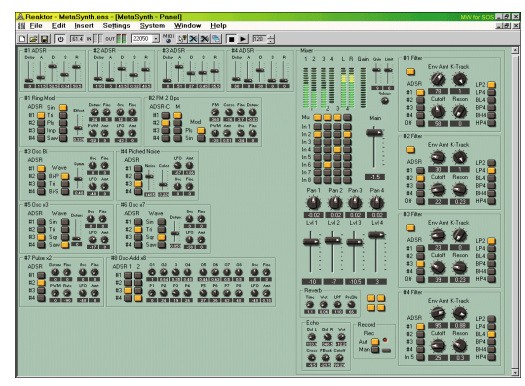

Fig.2 MidiSoft PlayPiano note identifies interface

\section{Necessity of multimedia technology for teaching piano Universities}

In the traditional piano teaching model, teachers focus more on teaching students theoretical knowledge and to be properly piano model, teachers speak more, practice less. Teaching model of a single, rely mainly on teacher verbal narrated, often appear Tang guan phenomenon. Lead to depression and classroom atmosphere relatively low, student interest can not be effectively mobilized. Therefore, in this boring classroom environment, although very hard to teach the teachers, but the students' practical learning effect is not satisfactory, low teaching efficiency. Over time, it will have a serious negative impact on student learning piano, as well as its future development. Above it can be described as shortcomings of the traditional classroom teaching. However, the conventional piano teaching is one to one teaching. The teaching model with respect to the large classroom teaching, it would appear to be relatively flexible, efficient the. Teachers can based on the specific circumstances of the students, and carry out relevant education and training, it can be described as all its talent, Shi to teach. However, this kind of teaching mode, in piano teaching piano teaching in universities is not in line with China's "water and soil." 
Our country is a big country of education, especially in college enrollment in recent years, leading to the rapid expansion of university students; the class capacity is relatively large. However, resources are limited but teachers, one teaching mode to implement in piano teaching universities, apparently high cost, not reality. To sum up, there are many contradictions and problems in the current piano teaching in universities. Meanwhile, with the development of society, increasingly fierce competition for talent, talent for music made a higher demand. For this reason, the traditional mode of education necessary to reform the piano, innovative teaching methods, improve teaching effectiveness. However, with the development of multimedia technology, its functions increasingly powerful and diverse, flexible coupled with freedom, vivid, educational advantages beyond time and space, can effectively solve the many contradictions appearing in the above teaching piano and piano teaching universities multimedia imperative.

\section{The impact of multimedia on the piano teaching reform}

Enrich teaching methods to improve the current interest in learning, teaching piano universities, lecture-style teaching methods still occupy a major position. Teachers play the role of preaching in the teaching process. Teaching is totally dependent on textbooks, set the appropriate teaching objectives based on the old text, and piano teaching. Aids are not only relatively simple, and quite scarce. Above the classroom, teachers teach the way of verbal teaching particular theory; there are often drawbacks chalk and talk. For some abstract theoretical knowledge, teachers simply by virtue of the language is difficult to enabling students to easily understand and accept, poor efficiency of student learning.

Multimedia technology used in piano teaching, to achieve a revolutionary education reforms. Piano education provides more tools and information, greatly improved the hardware environment piano education. Multimedia technology in teaching piano, can fully mobilize the language, text, sound and video images for the teaching of the services and other factors. Teachers according to the teaching objectives and teaching content, collection on network-related teaching materials, the mobilization and combination of the above factors, making vivid, lifelike courseware, and then specifically to teaching. Combining auditory, visual, tactile and other educational resources, the students multi-sensory stimulation, so abstract and difficult theoretical knowledge embodied by the difficult simplified, so as to facilitate the students' understanding and acceptance.

In addition, multimedia devices applications, making classroom teaching to become pan and Mao, a lively, can effectively mobilize the students' interest and enthusiasm, it allows students to actively participate in the teaching, become the subject of learning. In such a classroom teaching, students' learning efficiency is relatively high, is not only conducive to solve difficult problems, but also to deepen the impression of their students what they have learned.

\section{Realization of interactive teaching, diversity and individuality of the same}

Multimedia technology in teaching piano, piano teaching can liberate the productive forces to a large extent, to improve the quality of education and to optimize the piano plays an important role. To effectively activate its communication between teachers and students, "teaching" and "learning" interactive multimedia teaching piano most outstanding contribution. Thereby enhancing the student learning initiative and $\mathrm{AC}$ type, improve the teaching of enlightening and interesting to realize the intuitive and vivid knowledge. Multimedia piano collective teaching is not only a traditional classroom productivity advantages, but also combines the efficiency of one teaching. Thus, the use of multimedia can effectively improve the interaction between teachers and students, to achieve the same diversity and individuality.

Internet is a huge treasure trove of resources, during which contains a wealth of piano education resources. Whether a student or teacher, will find in this repository to the educational resources they need, to download and save. Further related studies are a timely manner through advanced electronics and multimedia devices. Students range in learning, but also not limited to the school being; it is not limited to learning during class time, a detailed study and appreciation gossip using the corresponding multimedia electronic devices. Thus, the use of multimedia technology is piano teaching; effective 
solution is the constraints of time students, making the piano learning more freedom, more favorable learning environment.

\section{Multimedia and multimedia technology}

Translated from the English word multimedia, Multimedia and media is a composite of multiple two words together. Media (medium) is the carrier of information, the exchange of information between people is an intermediary, the original two meanings: one refers to the storage of information entities, such as disks, optical disks, magnetic tape, semiconductor memory, etc., Chinese often translated as "medium "; the second refers to the transmission of information carriers, such as numbers, text, sound, graphics, etc., Chinese translated as" medium ", and the information most essential feature is a surface property characteristic of objective things, its manifestations are diverse, Thus, multimedia refers to various types of information integrated.

Multimedia technology is integration, technology and real-time interactive computer integrated processing sound Ventura information. Simply, that is able to support a variety of information and a variety of bank information (media) on the (media) is processed on the technology. Including media processing and coding technology, multimedia systems, multimedia information organization and management technology, multimedia communication network technology, multimedia and virtual reality machine interface technology, multimedia applications, multimedia synchronization technology, multimedia operating system technology, multimedia middleware technology multimedia switching technology, multimedia database technology, hypermedia technology, content-based retrieval technology, multimedia communication QoS management, multimedia conferencing systems, multimedia and video-on-demand and interactive TV technology, virtual reality space technologies.

General multimedia system consists of several hardware, operating system and software and other components, including computer hardware, all kinds of I / O, processing of multimedia information boards and other specialized disk reading device for the operating system is to mobilize hardware and run the software platform, the software is a tool to deal with specific tasks can be divided into the development of software and application software.

Multimedia technology has the following main features:

Comprehensive: Integrated management and control of information, and multi-channel fast read, store and synthesis.

Nonlinearity: nonlinear characteristics of multimedia technology changed the traditional sequential of read-write mode. Read and write the way people in the past mostly by frame, section, page, and progressive access to knowledge, and multimedia technology by method hypertext link (Hyper Text Link), and the content in a more flexible and presented in a way change to the reader.

Real-time: when the user gives the command, the corresponding multimedia information is able to get real-time control.

Interactive: it can form interaction between people and machines, people and machinery between the mutual exchange of operating environment and immersive scenes, people needed to control it. Human-computer interaction is the largest multimedia features.

Convenience: not only according to their own needs, interests, mission requirements, preferences and cognitive characteristics to the use of information, access to information, but also according to their own purposes and cognitive features reorganize, add, delete or modify information.

\section{Advantages of multimedia used in teaching}

Since entering the nineties, the rapid rise of multimedia technology to flourish, its application has spread to every corner of the national economy and social life, it is the human mode of production, work and even a huge lifestyle change. Similarly, the multimedia technology of the traditional teaching methods also has a huge influence and role. Now our universities are basically implemented a teaching model of traditional and multimedia teaching combination, compared with the traditional mode of teaching, multimedia teaching has a unique advantage, in the past a lot of formulas in the image of the traditional teaching model can not explain, principles, law, today you can get a clear performance, even some event or phenomenon difficult to observe under normal, educators and demonstration still be 
simulated with the help of computers. Vivid, rich picture, beautiful melody effect, making multimedia almost can go deep into people's thinking, in all areas of actions of love. Because multimedia has maps, text, sound, and Mao characteristics such as having many for education, the teaching process is particularly valuable features and functions, these features and functions are the other single media (such as slides, projection, film, sound recording, video, TV, etc.) do not have or do not completely have, therefore, as the traditional teaching model of multimedia teaching powerful supplement and improve, get everyone's consensus, which is, from the rapid development of multimedia teaching software and hardware and all of electronic teaching resources greatly enriched, it is also evident.

\section{Summary}

Advanced multimedia technology to break the limitations of traditional teaching is a wealth of Internet information to make piano teaching college easier, efficient and happy. More adapted to the requirements is the new era of music talents. Multimedia technology provides us with a good teaching platform, we clung to the pace of the times, give full play to the role of multimedia in teaching piano, with the vision to develop innovative music education, so that future multimedia technology and better teaching piano fused together, the two complement each other and strive to cultivate a more high-quality musical talent.

\section{References}

[1] Li Xiaowei. Application examples computer audition teaching ear training. Huang Zhong. 2000, 52-56.

[2] Luo Hailing. Using a computer in audition ear training courses in teaching. Artistic exploration, 2000, 12-17.

[3] CONSERVATORY. Multimedia CD-ROM technology and the future of music materials. Music. In March, 2000, 34-37.

[4] Chen Ya first. On the Music Education Reform Solfeggio professional learning methods. Fujian Normal University (Philosophy and Social Sciences). 2001,56-59.

[5] Zhang Lan. Solfeggio Teaching in Universities explore new models - computer music system technology and computer multimedia system in Solfeggio Teaching in Universities. Yunnan Art College, 2002(4), 124-127.

[6] Yan sharp. The necessity of the use of computer technology to aid in the teaching ear training teaching. Shenyang Conservatory of Music. 2002, 91-95.

[7] Kongya Lei. Teachers Solfeggio multimedia teaching aid application. People music. 2003, 11-15.

[8] Houbao Min. Solfeggio teaching of multimedia software applications. Hulunbeier College. 2005 , 57-60. 